PERSPECTIVE

\title{
Patient-Reported Outcomes in Clinical Trials of Rare Diseases
}

\author{
Ethan Basch, $M D^{1,2}$ and Antonia V. Bennett, $P h D^{2}$
}

${ }^{1}$ Cancer Outcomes Research Program, Lineberger Comprehensive Cancer Center, Department of Medicine, University of North Carolina, Chapel Hill, NC, USA; ${ }^{2}$ Cancer Outcomes Research Program, Lineberger Comprehensive Cancer Center, Department of Health Policy and Management, University of North Carolina, Chapel Hill, NC, USA.

The science of measuring patient-reported outcomes (PROs) has advanced substantially in recent decades, allowing evaluation of how patients feel and function in clinical research. Assessment of the patient experience in populations with rare diseases can be successfully achieved using PRO measures when careful planning and rigorous methods are employed. A number of challenges exist when designing and implementing PRO analyses in rare disease contexts, including heterogeneity of outcomes, availability of suitable measures, recruitment, and selection of appropriate data collection methods. Strategies to address these exist and have been employed in past clinical research, particularly in pediatric populations. PRO assessments in rare disease clinical trials have been particularly successful through partnerships between investigators, PRO methodologists, and patient organizations. The overall goal of PRO measurement is to understand the patient experience and it provides an essential part of evaluating the impact of disease and treatment.

KEY WORDS: patient-reported outcome; rare disease; quality of life. J Gen Intern Med 29(Suppl 3):S801-3

DOI: $10.1007 / \mathrm{s} 11606-014-2892-\mathrm{Z}$

(c) Society of General Internal Medicine 2014

T $\mathrm{n} 2011$, ruxolotinib was approved by the U.S. Food and 1 Drug Administration (FDA) and European Medicines Agency (EMA) for use by patients with myelofibrosis, a rare myeloproliferative neoplasm characterized by cytopenias and marked splenomegaly. ${ }^{1}$ Investigators at the company developing the drug recognized early that symptoms related to the disease were common and often debilitating. Working with academic collaborators, the company supported primary qualitative studies in the patient population to determine which symptoms were most salient, and then supported creation and testing of a questionnaire to assess these symptoms. ${ }^{2,3}$ This process was conducted with serial consultations with the FDA and EMA to assure that the methods were satisfactory for a potential labeling claim of symptomatic benefits.

In the ruxolotinib pivotal phase 3 clinical trial, about 300 patients were randomly assigned to receive study drug or placebo. Every patient was given a smartphone with which they reported symptoms daily using a 0-10 numerical rating

Published online July 17, 2014 scale for each, assessing the worst intensity over the past 24 h. ${ }^{1}$ Compliance was more than 95\%. Results were striking: $45 \%$ of patients receiving the study drug experienced a $50 \%$ or greater improvement in symptom score compared to $5 \%$ in the placebo group. The drug was approved and symptom improvement was included in the label. The cost of the entire process of developing, integrating, and analyzing the symptom data accounted for less than $3 \%$ of the overall program budget.

A "patient-reported outcome" or "PRO" has become the preferred terminology for a data element directly reported by patients or their surrogates about experiences with care and preferences, including symptoms, functional status, or quality of life. ${ }^{4}$ Multiple entities have produced guidance documents offering standards for developing, assessing, implementing, and analyzing PROs, including regulatory agencies like the FDA, ${ }^{5}$ publishing standards organizations like CONSORT, ${ }^{6}$ quality standards organizations such as the National Quality Forum (NQF), ${ }^{7,8}$ and professional associations like the International Society for Quality of Life Research (ISOQOL) ${ }^{9}$ and the International Society for Pharmacoeconomics and Outcomes Research (ISPOR). ${ }^{10}$

The growing number of organizations representing the interests of people with rare diseases understandably includes a focus on how people feel and function, which requires measurement with PRO tools. Highly motivated populations and advocacy organizations can facilitate collection of patient-reported information. The example of ruxolitinib is informative, as the high compliance rate reflects patients' willingness and enthusiasm to participate in data generation. Nonetheless, PROs are still omitted from many trials, and therefore do not appear in the publications of trial results or drug labels, particularly in evaluations of rare diseases. ${ }^{11,12}$

However, many of the challenges presented by assessing PROs in rare diseases can be addressed with careful planning and by drawing on established and current PRO research. The much smaller patient population is a significant impediment to recruiting enough patients to clinical trials as well as to studies designed to develop or validate a PRO for that condition. ${ }^{13}$ In both cases, patient advocacy groups and clinical care networks are potential avenues for patient recruitment. For both rare and more common diseases, the primary challenge in assessing PROs 
in a clinical trial is identifying a questionnaire that has appropriate content and a feasible method of data collection for the patients and their disease. In June 2011, the EveryLife Foundation workshop on Clinical Evaluation of Rare Disease Treatments featured a practical, in-depth review of methods for selecting and adapting PROs for rare disease clinical trials. ${ }^{14}$

Nevertheless, it is not possible to collect PROs from all study participants. Observer-Reported Outcomes (ObsROs) may be used to assess observable symptoms and functioning when patients are too young, too ill, or have cognitive impairments that make them unable to respond to PRO survey questions. When patients are able to report their experience but have physical impairments to completing paper, computer, or automated phone surveys, PROs may be collected via an interviewer. ISPOR has published a guidance document for development and use of ObsROs and PRO assessment in pediatrics. ${ }^{15}$ Because many people with rare diseases are children, this is informing their development of recommendations for PRO assessment in rare disease clinical trials. ${ }^{16}$

If there is substantial heterogeneity in how the disease presents, there may not be discrete outcomes that are measureable across the population. A multi-attribute questionnaire may be used in such cases, as it was (successfully) in the case of ruxolitinib. Investigators with experience in PRO measure development and use must be engaged early in a research program. This may involve an academic collaboration, or hiring a PRO consulting firm, as the developers of ruxolitinib did.

Off-the-shelf questionnaires may not be appropriate or specific enough to measure the important outcomes in a particular rare disease, and considerable effort may be needed to develop and test PRO tools. Additionally, if a drug is being developed towards regulatory approval, discussion with the applicable agencies is necessary to assure the PRO measurement strategy is compatible with their standards. There has been a recent resurgence in the development of instruments that are concept specific (i.e., pain, fatigue, physical function) instead of disease specific, such as the National Institutes of Health (NIH) Patient Reported Outcomes Measurement Information System (PROMIS) measures, ${ }^{17}$ which is a boon for rare disease research because disease-specific measures are often not available.

Although challenges exist for assessing PROs when a condition is rare, many of the barriers are inherent to studying rare diseases rather than PRO measurement itself. The current body of research in PRO assessment methods and newly developed tools provide substantial knowledge and resources for rare disease clinical investigators. PRO assessments have been particularly successful through partnerships among clinical investigators, PRO methodologists, and patient organizations. Understanding how patients feel is essential information when characterizing a disease or a treatment. Arguably, without such information, our understanding of either one remains incomplete.

Conflict of Interest: The authors declare that they do not have a conflict of interest.

Corresponding Author: Ethan Basch, MD; Physician Office Building, University of North Carolina, 170 Manning Drive, Chapel Hill, NC 27516 (e-mail: ebasch@med.unc.edu).

\section{REFERENCES}

1. Verstovsek S, Mesa RA, Gotlib J, Levy RS, Gupta V, DiPersio JF, Catalano JV, Deininger M, Miller C, Silver RT, Talpaz M, Winton EF, Harvey JH Jr, Arcasoy MO, Hexner E, Lyons RM, Paquette R, Raza A, Vaddi K, Erickson-Viitanen S, Koumenis IL, Sun W, Sandor V, Kantarjian HM. A double-blind, placebo-controlled trial of ruxolitinib for myelofibrosis. N Engl J Med. 2012;366(9):799-807.

2. Mesa RA, Kantarjian $\mathbf{H}$, Tefferi A, et al. Validation of the serial use of the Myelofibrosis Symptom Assessment Form (MF-SAF) for measuring symptomatic improvement: performance in 86 myelofibrosis patients on INCB018424 clinical trial. Blood. 2009;114:3917-3917.

3. Mesa RA, Kantarjian H, Tefferi A, et al. Validation of the serial use of the Myelofibrosis Symptom Assessment Form (MF-SAF) for measuring symptomatic improvement: performance in 86 myelofibrosis patients on INCB018424 clinical trial. Blood. 2009;114:3917-3917.

4. Basch E. New Frontiers in Patient-Reported Outcomes: Adverse Event Reporting, Comparative Effectiveness, and Quality Assessment. Annu Rev Med. 2013 Nov 20. [Epub ahead of print].

5. U.S. Department of Health and Human Services, Food and Drug Administration. Guidance for industry: Patient-reported outcomes measures: Use in medical product development to support labeling claims. December 2009. Available at http://www.fda.gov/downloads/Drugs/ GuidanceComplianceRegulatoryInformation / Guidances / UCM193282.pdf (last accessed 6/5/13).

6. Calvert M, Blazeby J, Altman DG, Revicki DA, Moher D, Brundage MD, CONSORT PRO Group. Reporting of patient-reported outcomes in randomized trials: the CONSORT PRO extension. JAMA. 2013;309(8):814-22.

7. National Quality Forum. Patient-reported outcomes (PROs) in performance measurement. January 10, 2013. Available at: www.qualityforum.org/Projects/n-r/Patient-Reported_Outcomes/Patient-Reported_Outcomes.aspx, last accessed 4/26/14.

8. Basch E, Torda P, Adams K. Patient-reported outcomes-based performance measures. JAMA. 2013;310(2):139-40.

9. Brundage M, Blazeby J, Revicki D, Bass B, de Vet H, Duffy H, Efficace F, King M, Lam CL, Moher D, Scott J, Sloan J, Snyder C, Yount S, Calvert M. Patient-reported outcomes in randomized clinical trials: development of ISOQOL reporting standards. Qual Life Res. 2013;22(6):1161-75.

10. International Society for Pharmacoeconomics and Outcomes Research. ISPOR Good Practices for Outcomes Research Index. http:// www.ispor.org/workpaper/practices_index.asp, last accessed 4/26/14.

11. Gnanasakthy A, Mordin M, Clark M, DeMuro C, Fehnel S, CopleyMerriman C. A review of patient-reported outcome labels in the United States: 2006 to 2010. Value Health. 2012;15(3):437-42.

12. Brundage M, Bass B, Davidson J, Gueenan J, Bezjak A, Ringash J, Wilkinson A, Feldman-Stewart D. Patterns of reporting health-related quality of life outcomes in randomized clinical trials: implications for clinicians and quality of life researchers. Qual Life Res. 2011;20(5):653-64.

13. Leidy, N.K. Addressing Content Validity of PRO Measures: The Unique Case of Rare Diseases. EveryLife Foundation, Workshop 2: Clinical Evaluation of Rare Disease Treatments. June 14-15, 2011, Washington DC. http://www.everylifefoundation.org/wp-content/uploads/images/ workshopseries / 13-Leidy-Addressing-Content-Validity-of-PRO- 
Measures.pdf, http://www.ispor.org/workpaper/practices_index.asp, last accessed 4/26/14.

14. EveryLife Foundation for Rare Diseases. Workshop 2: Clinical Evaluation of Rare Disease Treatments, June 14-15, 2011 . http:// www.everylifefoundation.org/events/workshop-series/workshop-2/, last accessed 4/26/14.

15. Matza LS, Patrick D, Riley AW, et al. Pediatric patient-reported outcome instruments for research to support medical product labeling: Report of the ISPOR PRO good research practices for the assessment of children and adolescents task force. Value Health. 2013;16:461-79.
16. International Society for Pharmacoeconomics and Outcomes Research Patient- and Observer-Reported Outcomes (PROs and ObsROs) Measurement in Rare Disease Clinical Trials - Emerging Good Practices. Task Force Chair: Benjamin, K. http://www.ispor.org/TaskForces/ PROs-and-ObsROs-RareDisease-ClinicalTrials.asp Accessed April 26, 2014.

17. National Institute of Health, Patient Reported Outcome Measurement Information System. PROMIS $®$ Dynamic Tools to Measure Health Outcomes from the Patient Perspective. http://www.nihpromis.org Accessed April 26, 2014 